\title{
PAPER \\ Design Optimization of H-Plane Waveguide Component by Level Set Method
}

\author{
Koichi HIRAYAMA $^{\dagger a)}$, Yasuhide TSUJI ${ }^{\dagger}$, Shintaro YAMASAKI ${ }^{\dagger \dagger}$, Members , \\ and Shinji NISHIWAKI ${ }^{\dagger \dagger}$, Nonmember
}

\begin{abstract}
SUMMARY We present a design optimization method of H-plane waveguide components, based on the level set method with the finite element method. In this paper, we propose a new formulation for the improvement of a level set function, which describes shape, location, and connectivity of dielectric in a design region. Employing the optimization procedure, we demonstrate that optimized structures of an H-plane waveguide filter and T-junction are obtained from an initial structure composed of several circular blocks of dielectric.

key words: design optimization, level set method, adjoint variable method, finite element method, H-plane waveguide component
\end{abstract}

\section{Introduction}

Microwave devices have been developed with the advance of information communication technology. Structures of microwave devices are usually designed based on the improvement of conventional devices or on heuristic idea. Recently, the design of microwave devices based on optimization methods has also been reported, which is enabled by the advent of low-cost and high-powered computers. In H-plane waveguide components, a T-junction [1]-[3], a mode transformer [4], and a filter [5] have been designed using sizing and shape optimization methods. An optimal shape design of a waveguide-to-microstrip transition [6] and a waveguide transformer [7] has also been reported using the finite element method (FEM). A waveguide filter has been designed using the genetic algorithm [8].

In the field of structure analysis, in addition to sizing and shape optimization, topology optimization (TO) [9] and the level set method (LSM) [10] have been advanced, and recently they are applied to some optimization problems in the field of electromagnetic waves. In sizing and shape optimization, the location and shape of blocks of material in a design region are designed to maximize or minimize a specific objective function, while topology in a design region, such as the number and connectivity of blocks of material,

Manuscript received September 2, 2010.

Manuscript revised January 18, 2011.

${ }^{\dagger}$ The authors are with the Department of Electrical and Electronic Engineering, Kitami Institute of Technology, Kitami-shi, 090-8507 Japan.

${ }^{\dagger}$ The author is with the Department of Machinery and Control Systems, College of Systems Engineering and Science, Shibaura Institute of Technology, Saitama-shi, 337-8570 Japan.

${ }^{\dagger \dagger}$ The author is with the Department of Mechanical Engineering and Science, Graduate School of Engineering, Kyoto University, Kyoto-shi, 606-8501 Japan.

a)E-mail: hirakc@mail.kitami-it.ac.jp

DOI: 10.1587/transele.E94.C.874 remains fixed. TO and LSM involve the determination of features in a design region, such as the location, shape, number and connectivity of blocks of material, so that we could expect to obtain a novel configuration using TO and LSM.

By using TO, in the microwave frequency band, the design of a dielectric substrate in a patch antenna [11] and $\mathrm{H}$ plane waveguide component [12] have been reported, and in optical frequency band, a 90-degree bend of an optical waveguide [13] and two-dimensional optical periodic structure [14] have been presented. Also, in photonic crystal waveguides, the optimization of a bend [15]-[19], the improvement of radiation characteristic into free space [20], and the improvement of the coupling between a slab and photonic crystal waveguide [21] have been investigated.

On the other hand, LSM has been applied in the electromagnetic field to the maximization of a band gap in a photonic crystal [22] and the inverse scattering problem [23][26]. Also, in [27], LSM is applied to the design optimization of left-handed dielectric metamaterials which is composed by periodically located dielectric resonators in a cutoff waveguide, and the optimization method for resonant frequencies is presented, but that for reflection and/or transmission characteristics of the waveguide is not treated. Very recently, the shape optimized design of microwave dielectric resonators based on LSM has been reported [28]. However, since the shape optimization for a single block of dielectric is only conducted, and in addition, introductory description written in [10] is merely given as the formulation for LSM, the applicability to optimization problems including the number and connectivity of blocks of dielectric is not obvious.

In this paper, we present an optimization method on reflection and/or transmission characteristics of an H-plane waveguide component, based on LSM with FEM. In this optimization, however, it is highly difficult or impossible to determine as a function a small variation of a level set function, which describes shape, location, and connectivity of dielectric in a design region, so as to monotonically decrease an objective function, and so we propose an alternative formulation, where the small variation is determined as discrete values by using the same discretization as in FEM. It is an advantage that the discretization of the small variation is readily incorporated in FEM formulation. To confirm the validity of our formulation, we design an H-plane waveguide filter and T-junction, and demonstrate that optimized structures are obtained from an initial structure composed of 
several circular blocks of dielectric.

\section{Finite Element Analysis of H-Plane Waveguide Dis- continuity Problem}

We consider an H-plane waveguide discontinuity problem, as shown in Fig. 1, where boundary $\Gamma_{0}$ represents an electric wall and $\Gamma_{n}(n=1,2, \cdots, N)$ is the boundary between discontinuity region $\Omega$ and waveguide $n$. Here, we assume the field has no variation in the $z$ direction, and let $\phi=E_{z}\left(E_{z}\right.$ being the $z$ component of the electric field). Dividing region $\Omega$ into a number of linear rectangular elements shown in Fig. 2 and applying FEM, we obtain a final matrix equation as follows:

$$
[P]\{\phi\}=\{Q\}
$$

where the input and output port at $\Gamma_{n}$ is treated by employing the eigenfunction expansion with respect to the modes of waveguide $n$ [29]. After solving (1), we can compute the $S$ parameter in waveguide $n$ for the incidence of a propagating mode with unit amplitude from waveguide 1 as follows:

$$
S_{n 1}=-\delta_{n 1}+\left\{g_{n}\right\}^{T}\left\{\phi_{n}\right\}
$$

where $\delta_{n 1}$ represents the Kronecker delta, $\left\{g_{n}\right\}$ is a known vector related to a propagating mode in waveguide $n,\left\{\phi_{n}\right\}$ is a vector which has the values of $\phi$ on boundary $\Gamma_{n}$, and superscript $T$ denotes a transpose.

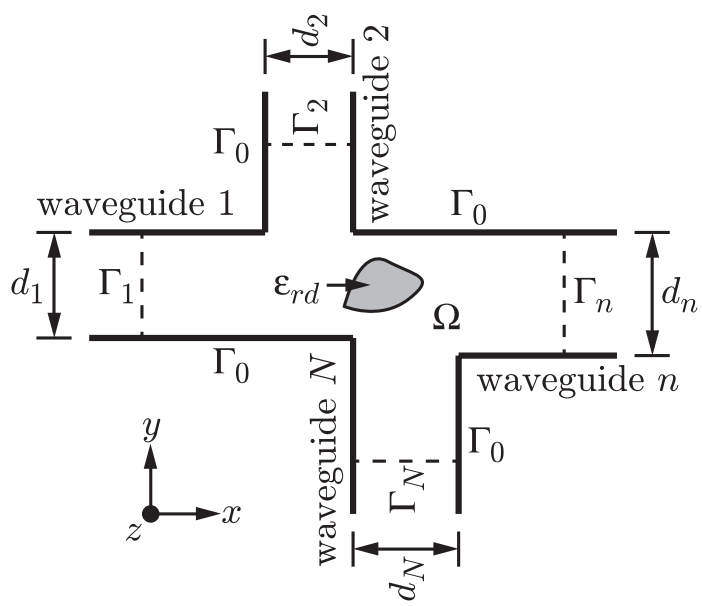

Fig. 1 Discontinuity in H-plane waveguide component

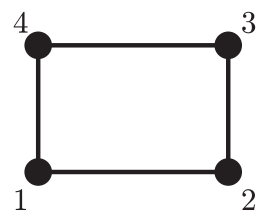

Fig. 2 Linear rectangular element.

\section{Formulation of Optimization Method Based on Level Set Method}

\subsection{Outline of Level Set Method}

For completeness of this paper, we briefly introduce LSM, which is described in detail in [10].

LSM determines interfaces between two materials, air and dielectric in this paper, in a design region through optimization procedure, and the interfaces are defined by the iso-contour of $w(\boldsymbol{r})=0$, where $w$ is a level set function and $\boldsymbol{r}$ is a location vector. In LSM, pseudo time $\tau$ is introduced, and the level set function is improved with the pseudo time so as to minimize a specific objective function under the following convection (or advection) equation known as the "level set equation":

$$
\frac{\partial w(\boldsymbol{r})}{\partial \tau}+V_{N}(\boldsymbol{r})|\nabla w(\boldsymbol{r})|=0
$$

where $V_{N}(\boldsymbol{r})$ stands for the component of velocity normal to the iso-contour of $w(\boldsymbol{r})$ and is determined from sensitivity analysis. The following signed distance function is usually adopted as a level set function:

$$
w(\boldsymbol{r})= \begin{cases}d(\boldsymbol{r}) & \text { for } \boldsymbol{r} \in \Omega_{\mathrm{air}} \\ 0 & \text { for } \boldsymbol{r} \in \partial \Omega \\ -d(\boldsymbol{r}) & \text { for } \boldsymbol{r} \in \Omega_{\text {dielectric }}\end{cases}
$$

where $\Omega_{\text {air }}$ and $\Omega_{\text {dielectric }}$ denote material regions of air and dielectric, respectively, and $\partial \Omega$ represents the interface between the two materials. Here $d(\boldsymbol{r})$ is an unsigned distance function defined as the distance from the point $\boldsymbol{r}$ to the interface $\partial \Omega$. Then the relative permittivity in a design region is represented using Heaviside function $H(w)$ as

$$
\varepsilon_{r}=\varepsilon_{r d}+\left(1-\varepsilon_{r d}\right) H(w)
$$

where $\varepsilon_{r d}$ denotes the relative permittivity of dielectric. Also, since $|\nabla w(\boldsymbol{r})|=1$ holds, (3) is solved using Euler method as follows:

$$
w^{(\tau+\Delta \tau)}(\boldsymbol{r})=w^{(\tau)}(\boldsymbol{r})-V_{N}^{(\tau)}(\boldsymbol{r}) \Delta \tau
$$

where $\Delta \tau$ is a time interval, and $w^{(\tau)}$ and $V_{N}^{(\tau)}$ represent the values of $w$ and $V_{N}$ at pseudo time $\tau$, respectively.

\subsection{Sensitivity Analysis}

Here the sensitivity is calculated using the adjoint variable method (AVM). Let $C$ be an objective function, which is minimized in an optimization problem, and in most cases of waveguide discontinuity problems $C$ is explicitly expressed with the absolute values of $\mathrm{S}$ parameters. Here we define $C$ as

$$
C=F\left(\left|S_{11}\right|,\left|S_{21}\right|, \cdots,\left|S_{N 1}\right|\right)
$$


where $F$ stands for a function with respect to $\left|S_{11}\right|,\left|S_{21}\right|, \cdots$, $\left|S_{N 1}\right|$. If the shape of the interfaces between dielectric and air is modified, the values of $\mathrm{S}$ parameters are changed. When the value of the level set function changes from $w(\boldsymbol{r})$ to $w(\boldsymbol{r})+\delta w(\boldsymbol{r})$ because of the modification of the shape of the interfaces, the sensitivity of the objective function $C$ is calculated as follows:

$$
\begin{aligned}
\delta C & =\sum_{n=1}^{N} \frac{\partial F}{\partial\left|S_{n 1}\right|} \operatorname{Re}\left(\frac{S_{n 1}^{*}}{\left|S_{n 1}\right|} \delta S_{n 1}\right) \\
& =\sum_{n=1}^{N} \frac{\partial F}{\partial\left|S_{n 1}\right|} \operatorname{Re}\left(\frac{S_{n 1}^{*}}{\left|S_{n 1}\right|}\left\{\tilde{g}_{n}\right\}^{T}\{\delta \phi\}\right)
\end{aligned}
$$

where Re stands for the real part of a complex number, a superscript $*$ indicates complex conjugate, and the sensitivity of $S_{n 1}, \delta S_{n 1}$, is obtained from (2). Here, $\left\{\tilde{g}_{n}\right\}$ is a vector whose components are the values of $\left\{g_{n}\right\}$ at the nodes on boundary $\Gamma_{n}$ and zero elsewhere. We can obtain the sensitivity of $\{\phi\},\{\delta \phi\}$, from (1)

$$
\{\delta \phi\}=-[P]^{-1}([\delta P]\{\phi\})
$$

where

$$
\begin{aligned}
& {[\delta P]\{\phi\}=-k_{0}^{2} \sum_{e} \iint_{e} \delta \varepsilon_{r}\{N\}\left(\{N\}^{T}\{\phi\}_{e}\right) d x d y} \\
& \delta \varepsilon_{r}=\left(1-\varepsilon_{r d}\right) \delta(w) \delta w
\end{aligned}
$$

Here $k_{0}$ is a wave number in vacuum, $\{N\}$ is a shape function vector of a rectangular element, $\{\phi\}_{e}$ is a vector which has the values of $\phi$ on the vertices of the element, $\iint_{e} d x d y$ represents the surface integral over the element, and $\sum_{e}$ denotes the summation over all the elements. The sensitivity of $\varepsilon_{r}, \delta \varepsilon_{r}$, is obtained from (5), and $\delta(w)$ is the Dirac delta function.

\subsection{Formulation of Velocity}

In LSM, one is required to formulate velocity $V_{N}$ in each of applications. For example, the velocity for an eigenvalue problem on photonic band gap is presented in Sect. 3 of [22], and that for an inverse problem on the determination of perfect conductor shape is in (11) of [30]. Here we propose a formulation of $V_{N}$ for the optimization of shape, location, and connectivity of dielectric in H-plane waveguide components, as described below.

From (6), we can set

$$
V_{N} \Delta \tau=-\delta w
$$

Substituting (9) to (11) into (8), the sensitivity of $C$ is expressed as

$$
\begin{aligned}
\delta C= & \sum_{n=1}^{N} \frac{\partial F}{\partial\left|S_{n 1}\right|} \operatorname{Re}\left[\frac{S_{n 1}^{*}}{\left|S_{n 1}\right|}\left\{\tilde{g}_{n}\right\}^{T}[P]^{-1} k_{0}^{2}\left(1-\varepsilon_{r d}\right)\right. \\
& \left.\sum_{e} \iint_{e} \delta(w) \delta w\{N\}\left(\{N\}^{T}\{\phi\}_{e}\right) d x d y\right]
\end{aligned}
$$

In the above equation, since it is highly difficult or impossible to determine $\delta w$ as a function so as to monotonically decrease the objective function, we propose an alternative formulation, in which $\delta w$ is determined as discrete values.

We discretize the value of $\delta w$ in a rectangular element as

$$
\delta w=\{N\}^{T}\{\delta w\}_{e}
$$

where $\{\delta w\}_{e}$ is a vector which has the values of $\delta w$ on the vertices of the element. From (9), (10), (11), and (14), we obtain

$$
\{\delta \phi\}=[P]^{-1}[W]\{\delta w\}
$$

where

$$
\begin{aligned}
{[W]=} & k_{0}^{2}\left(1-\varepsilon_{r d}\right) \\
& \sum_{e} \iint_{e}\left(\{N\}^{T}\{\phi\}_{e}\right) \delta(w)\{N\}\{N\}^{T} d x d y
\end{aligned}
$$

and $\{\delta w\}$ is a vector which has the values of $\delta w$ on the vertices of all the elements. Accordingly, the sensitivity of $C$ is expressed as

$$
\delta C=\sum_{n=1}^{N} \frac{\partial F}{\partial\left|S_{n 1}\right|} \operatorname{Re}\left(\frac{S_{n 1}^{*}}{\left|S_{n 1}\right|}\left\{\Phi_{n}\right\}^{T}[W]\right)\{\delta w\}
$$

where

$$
\left\{\Phi_{n}\right\}=\left([P]^{T}\right)^{-1}\left\{\tilde{g}_{n}\right\}
$$

We notice that $\delta C$ always has a negative value when we set the value of $\{\delta w\}$ as

$$
\{\delta w\}=-\alpha \sum_{n=1}^{N} \frac{\partial F}{\partial\left|S_{n 1}\right|} \operatorname{Re}\left(\frac{S_{n 1}^{*}}{\left|S_{n 1}\right|}[W]^{T}\left\{\Phi_{n}\right\}\right)
$$

where $\alpha$ is a positive number. When, using (12) and (19), $w(\boldsymbol{r})$ is improved with pseudo time $\tau$ in accordance with (6), the value of the objective function $C$ will decrease monotonically with $\tau$, and then be minimized. In iterations of optimization based on LSM, $\alpha$ should be chosen so as to satisfy $\max (|\delta w|)<\Delta$, where $\max (|\delta w|)$ represents the largest value of the absolute value over the components of $\{\delta w\}$, and $\Delta$ stands for the length of a side of a rectangular element.

\section{Numerical Examples}

\subsection{Waveguide Filter}

We consider an H-plane waveguide component of width $a=22.9 \mathrm{~mm}$, as shown in Fig. 3, where the $\mathrm{TE}_{10}$ mode incidence from boundary $\Gamma_{1}$ is assumed. Here we determine an optimal distribution of dielectric with relative permittivity $\varepsilon_{r d}=2$ in a design region of length $4 a$ so as to minimize the transmitted power at $10 \mathrm{GHz}$. Figure 3 also shows an initial structure for the optimization based on LSM, which has six circular dielectric posts with relative permittivity $\varepsilon_{r d}$ in the 


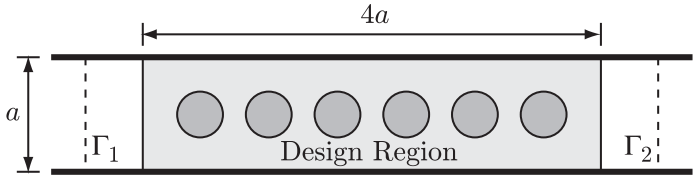

Fig. 3 Initial structure of H-plane waveguide component.

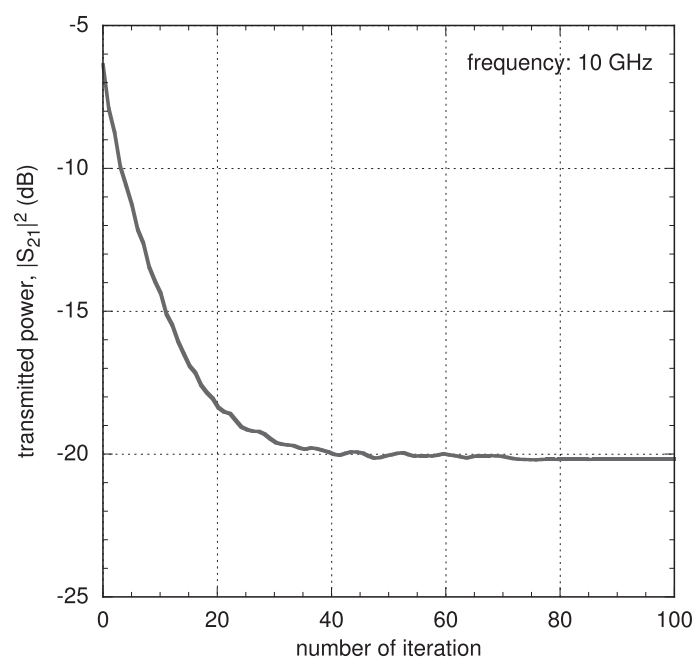

Fig. 4 Change of transmitted power versus the number of iteration in $\mathrm{H}$-plane waveguide component.

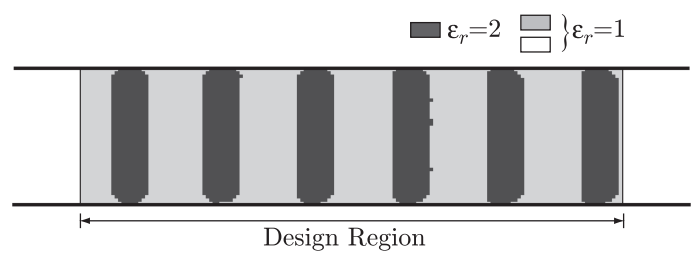

Fig. 5 Optimized structure in H-plane waveguide filter.

design region. In LSM, one can use any shape of dielectrics as an initial structure, and here we select circular posts so that the initial structure does not have any specific feature. In this case, the objective function is given as follows:

$$
\text { Minimize } C=\left|S_{21}\right|^{2} \text { at } 10 \mathrm{GHz}
$$

Figure 4 shows the transmitted power against the number of the iteration in LSM, and Fig. 5 shows the structure obtained after 100 iterations. We notice that a onedimensional filter is made up automatically by a onedimensional periodic structure whose period length is $d=$ $16.1 \mathrm{~mm}$ with dielectric thickness of $6.2 \mathrm{~mm}$. To explain the optimized structure, we show in Fig. 6 the attenuation constant $\alpha$ at $10 \mathrm{GHz}$ and the upper and lower limit of the stopband in an infinite periodic structure, which are estimated according to the formulation in the Appendix. In this figure the normalized attenuation constant has the maximum value at a period length $d=15.9 \mathrm{~mm}$ with dielectric width of $6.0 \mathrm{~mm}$, and we notice that these values and the upper and lower limit of the stopband are in accordance with those of the optimized structure.

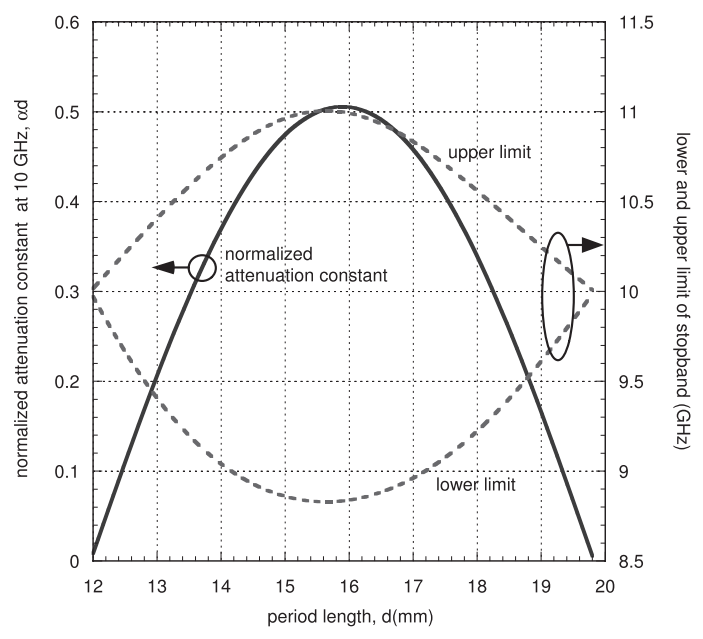

Fig. 6 Estimated values of attenuation constant, upper and lower limit of stopband in 1-D infinite periodic structure.

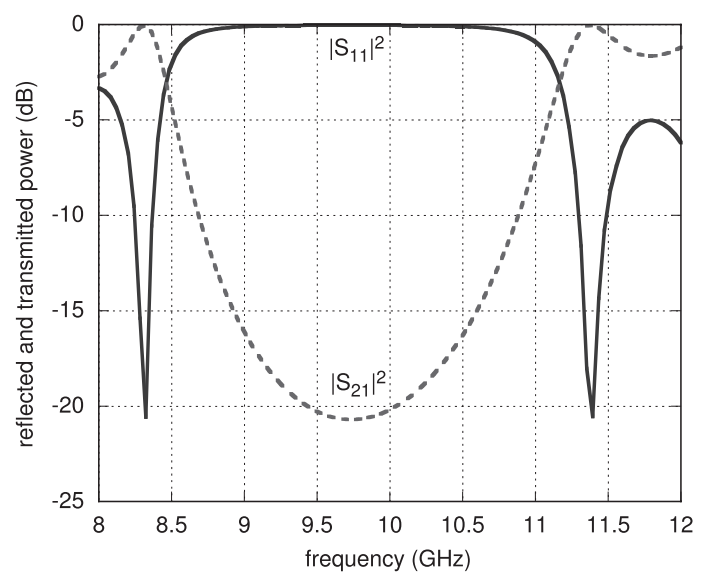

Fig. 7 Frequency characteristic of optimized structure in H-plane waveguide filter.

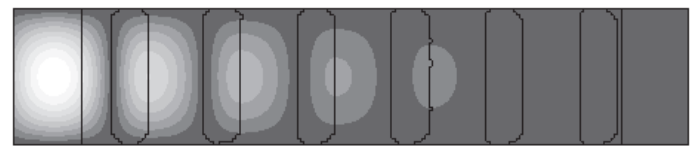

Fig. 8 Electric field intensity distribution of optimized structure in $\mathrm{H}$-plane waveguide filter at $10 \mathrm{GHz}$.

Figure 7 shows the frequency characteristic of the optimized structure. We find that this structure realizes a filter which has the transmitted power of less than $-20 \mathrm{~dB}$ in the frequency between 9.5 and $10.0 \mathrm{GHz}$. Also, Fig. 8 shows the electric field intensity distribution at $10 \mathrm{GHz}$, and one can observe the reflection by the periodic structure.

To investigate the relation between an initial and optimized structure, we performed the optimization for an initial structure of five, seven, or eight circular dielectric posts and obtained as an optimized structure a one-dimensional periodic structure whose periodic length and dielectric thickness are the same as for an initial structure of six posts, but the number of dielectrics in the optimized structures are five for 


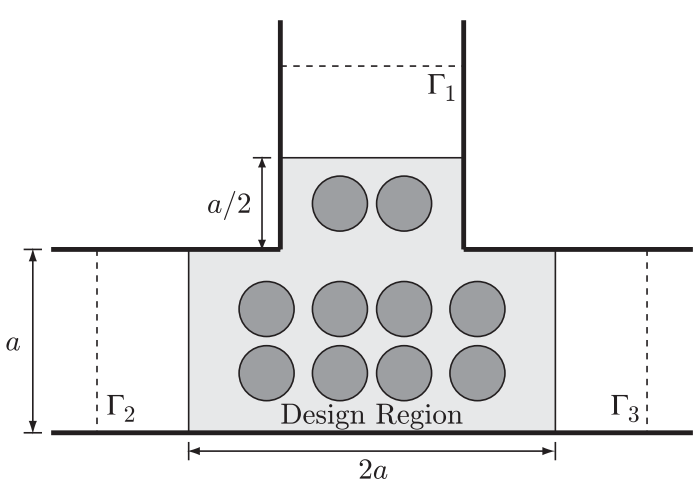

Fig. 9 Initial structure of H-plane T-junction.

five or eight posts and six for seven posts. Since dielectrics do not appear newly (may disappear or join each other) through the optimization by a standard LSM, it is very natural that the optimized structure composed of five dielectrics is made up for an initial structure of five posts. It is desirable that an optimized structure of six dielectrics is made up for an initial structure of more than six posts, because the the transmitted power at $10 \mathrm{GHz}$ is about $-20 \mathrm{~dB}$ in six dielectrics, while it rises to about $-16 \mathrm{~dB}$ in five dielectrics due to the decrease of the number of periods. However, both optimized structures composed of five and six dielectrics are capable as local minima in the optimization, because the length of the design region of $4 a=91.6 \mathrm{~mm}$ corresponds to about 5.7 times periodic length of $d=16.1 \mathrm{~mm}$. Anyway, it is necessary to perform the optimization for some initial structures, because it is difficult to avoid falling into a local minimum in a standard LSM.

\subsection{Optimization of T-Junction}

Next, we consider an H-plane T-junction of width $a=$ $22.9 \mathrm{~mm}$, as shown in Fig. 9, where the $\mathrm{TE}_{10}$ mode incidence from boundary $\Gamma_{1}$ is assumed. Here we determine an optimal distribution of dielectric with relative permittivity $\varepsilon_{r d}=2$ in a design region of the junction added by length $a / 2$ into waveguides 1 to 3 so as to minimize the reflected power $\left|S_{11}\right|^{2}$ and divide the transmitted power into waveguides 2 and 3 with the power ratio $2: 1$ over 9 to $11 \mathrm{GHz}$. In this case, the objective function is given as follows:

$$
\begin{gathered}
\text { Minimize } \quad C=\sum_{f_{m}}\left[\left|S_{11}\right|^{2}+\left(\left|S_{21}\right|-\sqrt{2}\left|S_{31}\right|\right)^{2}\right] \\
\text { at } f_{m}=9,10,11 \mathrm{GHz}
\end{gathered}
$$

Figure 10 shows the reflected power, $\left|S_{11}\right|^{2}$, and the transmitted power, $\left|S_{21}\right|^{2}$ and $\left|S_{31}\right|^{2}$, against the number of the iteration in LSM, and Fig. 11 shows the structure obtained after 100 iterations. The frequency characteristic for the optimized structure is shown in Fig. 12. We observe that the transmitted powers in waveguides 2 and 3 are approximately constant over 9 to $11 \mathrm{GHz}$ and the power ratio of them is $1.99: 1$ at $10 \mathrm{GHz}$, while the reflected power is less than $-23.5 \mathrm{~dB}$. These values are improved in comparison

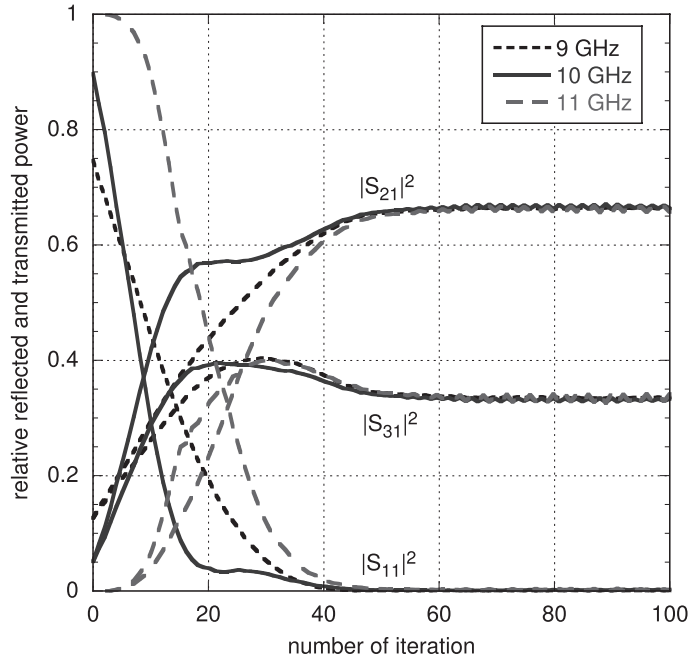

Fig. 10 Change of reflected and transmitted power versus the number of iteration in $\mathrm{H}$-plane T-junction.

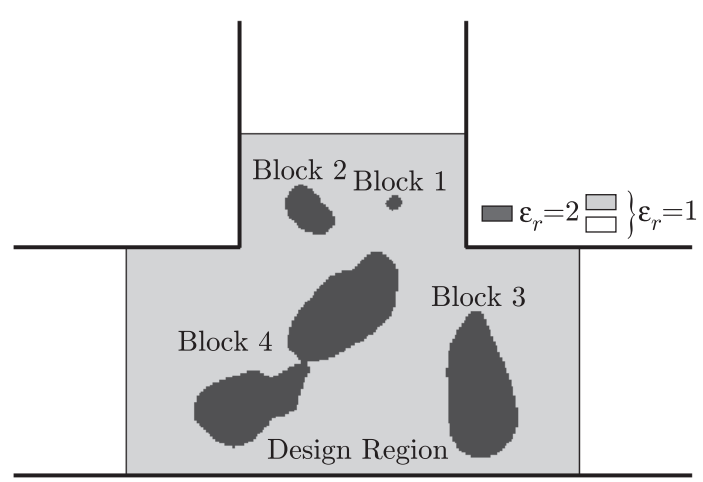

Fig. 11 Optimized structure in H-plane T-junction.

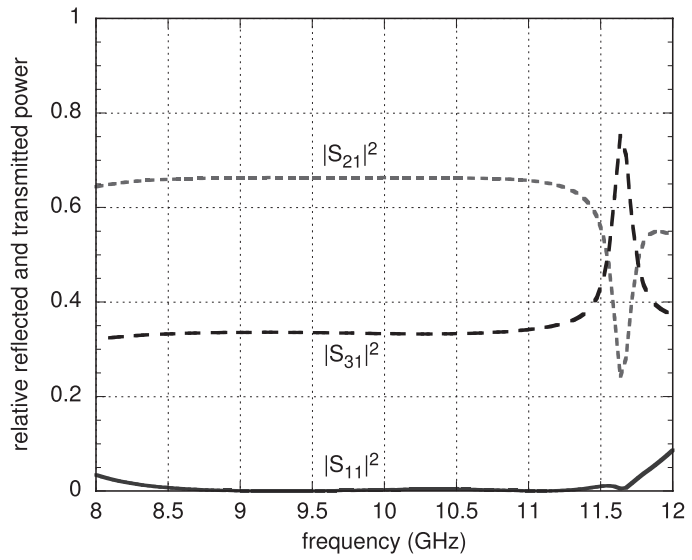

Fig. 12 Frequency characteristic of optimized structure in H-plane T-junction.

with those of TO in [12], which are $1.8: 1$ and $-18.5 \mathrm{~dB}$, respectively. The main reason is that, in the optimized structure of TO, it is essential to simplify the obtained structure since materials of intermediate permittivity between air and dielectric are contained, while, in the optimized structure 


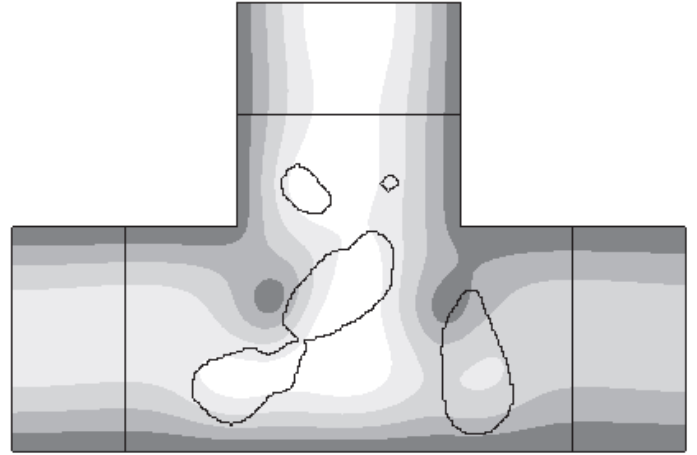

Fig. 13 Electric field intensity distribution for optimized structure in $\mathrm{H}$-plane T-junction at $10 \mathrm{GHz}$.

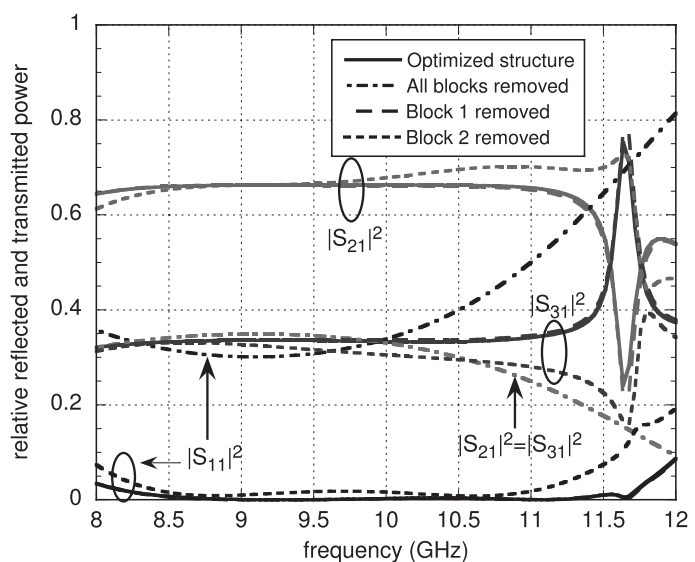

Fig. 14 Frequency characteristic of optimized structure, one with all blocks removed, and one with block 1 or 2 removed.

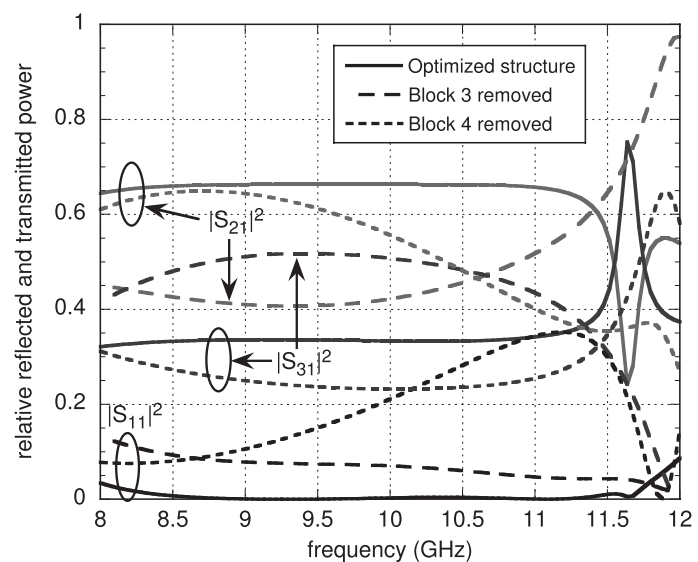

Fig. 15 Frequency characteristic of optimized structure and one with block 3 or 4 removed.

of LSM, the simplification is not required since dielectrics have clear shape.

For convenience of explanation, we name each of the dielectrics block $n(n=1,2,3,4)$, as shown in Fig. 11. Figure 13 shows the electric field intensity distribution for the optimized structure in an $\mathrm{H}$-plane T-junction at $10 \mathrm{GHz}$. It seems that block 4 guides the incident wave from waveguide
1 to waveguide 2, and that block 3 reflects part of the incident wave reflected at the waveguide wall to the direction of waveguide 2. Anyway, without optimization methods, it is difficult to determine appropriately the size and location of these blocks. To investigate a role of each of the dielectric blocks in detail, we compute the frequency characteristic of optimized structures with one of the dielectric blocks removed. Figures 14 and 15 show the frequency characteristic of the optimized structure and one with block 1, 2, 3, or 4 removed. In Fig. 14, the frequency characteristic of the structure with all the blocks removed is also shown, and the power is divided equally into waveguides 1,2 , and 3 at $10 \mathrm{GHz}$. Block 1 is very small, and the frequency characteristic of the optimized structure with block 1 removed is almost the same as that of the optimized structure. Block 2 is relatively small, but we notice that block 2 is necessary to keep the power ratio divided into waveguides 2 and 3 over 9 to $11 \mathrm{GHz}$. Block 4 prevents the reflection to waveguide 1 and guides the incident wave to waveguide 2 over 9 to $11 \mathrm{GHz}$, but, if block 3 is removed and it does not reflect the wave to the direction of waveguide 2, the transmitted power into waveguide 2 becomes less than that of the optimized structure, and that into waveguide 3 increases.

The H-plane T-junction was divided into 40000 linear rectangular elements, and the number of the unknowns in (1) was 39798. The computational time on a PC with Pentium 4 of $3.2 \mathrm{GHz}$ and $2 \mathrm{~GB}$ memory was about 15 minutes for 100 iterations in the T-junction.

\section{Conclusion}

We presented an optimization method on reflection and/or transmission characteristics of an H-plane waveguide component, based on LSM with FEM. Here we proposed a formulation in LSM, where a small variation of the level set function, $\delta w$, is determined as discrete values, not as a function, by using the same discretization as in FEM. To confirm the validity of our formulation, we illustrated through some numerical examples that an optimized structure is obtained from an initial structure composed of several circular blocks of dielectric.

The proposed formulation for the discretization of $\delta w$ is versatile, and so our optimization method based on LSM with FEM will be readily extended and applied to optimization on reflection and/or transmission characteristics in other electromagnetic and optical waveguides, including threedimensional ones.

\section{References}

[1] H.B. Lee, H.K. Jung, and S.Y. Hahn, "Shape optimization of Hplane waveguide tee junction using edge finite element method," IEEE Trans. Magn., vol.31, no.3, pp.1928-1931, May 1995.

[2] Y.S. Chung, J. Ryu, C. Cheon, I.H. Park, and S. Hahn, "Optimal design method for microwave device using time domain method and design sensitivity analysis - Part I: FETD case," IEEE Trans. Magn., vol.37, no.5, pp.3289-3293, Sept. 2001.

[3] Y. Yang, T. Halleöd, D. Ericsson, A. Hellervik, A. Bondeson, and T. 
Rylander, "Gradient optimization of microwave devices using continuum design sensitives from the adjoint problem," IEEE Trans. Magn., vol.41, no.5, pp.1780-1783, May 2005.

[4] M.C. Yang, J.H. Li, and K.J. Webb, "Functional waveguide mode transformers," IEEE Trans. Microw. Theory Tech., vol.52, no.1, pp.161-169, Jan. 2004

[5] P. Arcioni, M. Bozzi, M. Bressan, G. Conciauro, and L. Perregrini, "Fast optimization, tolerance analysis, and yield estimation of $H-/ E$ Plane waveguide components with irregular shapes," IEEE Trans. Microw. Theory Tech., vol.52, no.1, pp.319-328, Jan. 2004.

[6] H.B. Lee and T. Itoh, "A systematic optimum design of waveguideto-microstrip transition," IEEE Trans. Microw. Theory Tech., vol.45, no.5, pp.803-809, May 1997.

[7] D. Nair and J.P. Webb, "Optimization of microwave devices using 3-D finite elements and the design sensitivity of the frequency response," IEEE Trans. Magn., vol.39, no.3, pp.1325-1328, May 2003.

[8] M. Ohira, H. Deguchi, M. Tsuji, and H. Shigesawa, "Novel waveguide filters with multiple attenuation poles using dual-behavior resonance of frequency-selective surfaces," IEEE Trans. Microw. Theory Tech., vol.53, no.11, pp.3320-3326, Nov. 2005.

[9] M.P. Bendsøe and O. Sigmund, Topology Optimization: Theory, Methods and Applications, Springer, New York, 2004.

[10] S. Osher and R. Fedkiw, Level Set Method and Dynamic Implicit Surfaces, Springer, New York, 2003

[11] G. Kiziltas, D. Psychoudakis, J.L. Volakis, and N. Kikuchi, "Topology design optimization of dielectric substrates for bandwidth improvement of a patch antenna," IEEE Trans. Antennas Propag., vol.51, no.10, pp.2732-2743, Oct. 2003.

[12] K. Hirayama, Y. Tsuji, T. Nomura, K. Sato, and S. Nishiwaki, "Application of topology optimization to H-plane waveguide component," IEICE Trans. Electron., vol.E90-C, no.2, pp.282-287, Feb. 2007.

[13] Y. Tsuji, K. Hirayama, T. Nomura, K. Sato, and S. Nishiwaki, "Design of optical circuit devices using topology optimization method with function-expansion-based refractive index distribution," IEEE Photonics Technol. Lett., vol.20, no.12, pp.982-984, June 2008.

[14] T. Nomura, S. Nishiwaki, K. Sato, and K. Hirayama, "Topology optimization for the design of periodic microstructures composed of electromagnetic materials," Finite Elements in Analysis and Design, vol.45, no.3, pp.210-226, Feb. 2009.

[15] J.S. Jensen and O. Sigmund, "Systematic design of photonic crystal structures using topology optimization: Low-loss waveguide bends," Appl. Phys. Lett., vol.84, no.12, pp.2022-2024, March 2004.

[16] P.I. Borel, A. Harpøth, L.H. Frandsen, M. Kristensen, P. Shi, J.S Jensen, and O. Sigmund, "Topology optimization and fabrication of photonic crystal structures," Opt. Express, vol.12, no.9, pp.19962001, May 2004.

[17] L.H. Frandsen, A. Harpøth, P.I. Borel, M. Kristensen, J.S. Jensen, and O. Sigmund, "Broadband photonic crystal waveguide $60^{\circ}$ bend obtained utilizing topology optimization," Opt. Express, vol.12, no.24, pp.5916-5921, Nov. 2004.

[18] J.S. Jensen, O. Sigmund, L.H. Frandsen, P.I. Borel, A. Harpøth, and M. Kristensen, "Topology design and fabrication of an efficient double $90^{\circ}$ photonic crystal waveguide bend," IEEE Photonics Technol. Lett., vol.17, no.6, pp.1202-1204, June 2005.

[19] J.S. Jensen and O. Sigmund, "Topology optimization of photonic crystal structures: A high-bandwidth low-loss T-junction waveguide," J. Opt. Soc. Am. B, vol.22, no.6, pp.1191-1198, June 2005.

[20] W.R. Frei, D.A. Tortorelli, and H.T. Johnson, "Topology optimization of a photonic crystal waveguide termination to maximize directional emission," Appl. Phys. Lett., vol.86, 111114, March 2005.

[21] K. Hirayama, Y. Tabata, Y. Tsuji, and Y. Hayashi, "Topology optimization of coupling between slab and photonic crystal waveguide,' Microw. Opt. Technol. Lett., vol.50, no.12, pp.3009-3012, Dec. 2008

[22] C.Y. Kao, S. Osher, and E. Yablonovitch, "Maximizing band gaps in two-dimensional photonic crystals by using level set methods," Appl. Phys. B, vol.81, no.2-3, pp.235-244, July 2005.

[23] A. Litman and K. Belkebir, "Two-dimensional inverse profiling problem using phaseless data," J. Opt. Soc. Am. A, vol.23, no.11, pp.2737-2746, Nov. 2006.

[24] M. Benedetti, M. Donelli, D. Lesselier, and A. Massa, "A two-step inverse scattering procedure for the qualitative imaging of homogeneous cracks in known host media - Preliminary results," IEEE Antennas Wireless Propag. Lett., vol.6, no.1, pp.592-595, 2007.

[25] D.A. Woten, M.R. Hajihashemi, A.M. Hassan, and M. El-Shenawee, "Experimental microwave validation of level set reconstruction algorithm," IEEE Trans. Antennas Propag., vol.58, no.1, pp.230-233, Jan. 2010.

[26] M. Schweiger, O. Dorn, A. Zacharopoulos, I. Nissilä, and S.R Arridge, "3D level set reconstruction of model and experimental data in diffuse optical tomography," Opt. Express, vol.18, no.1, pp.150-164, Jan. 2010.

[27] S. Yamasaki, T. Nomura, K. Sato, N. Michishita, and Y. Yamada, "Design optimization of left-handed dielectric metamaterial using a level set based structural optimization method," IEICE Technical Report, A.P2009-128, Nov. 2009.

[28] H. Khalil, S. Bila, M. Aubourg, D. Baillargeat, S. Verdeyme, F. Jouve, C. Delage, and T. Chartier, "Shape optimized design of microwave dielectric resonators by level-set and topology gradient methods," Int. J. RF and Microw. Comp.-Aided Eng., vol.20, no.1, pp.33-41, Jan. 2010.

[29] J. Jin, The Finite Element Method in Electromagnetics, 2nd ed. Sect. 11, Wiley-IEEE Press, New York, 2002.

[30] R. Ferrayé, J.-Y. Dauvignac, and C. Pichot, "An inverse scattering method based on contour deformations by means of a level set method using frequency hopping technique," IEEE Trans. Antennas Propag., vol.51, no.5, pp.1100-1113, May 2003.

[31] R.E. Collin, Field Theory of Guided Waves, 2nd ed., Ch.9, IEEE Press, New York, 1991.

\section{Appendix: 1-D Infinite Periodic Structure}

The eigenvalue equation in a stopband of a 1-D infinite periodic waveguide as shown in Fig. A. 1 is given as follows [31]:

$$
\cosh \alpha d=\left|\frac{A_{11}+A_{22}}{2}\right|
$$

where $\alpha$ and $d$ are an attenuation constant and period length of the periodic waveguide, respectively, and $A_{11}$ and $A_{22}$ stand for the components of the wave-amplitude transmission matrix for a period of the waveguide. When we represent the wave-amplitude transmission matrix by using only $\mathrm{TE}_{10}$ mode, we obtain

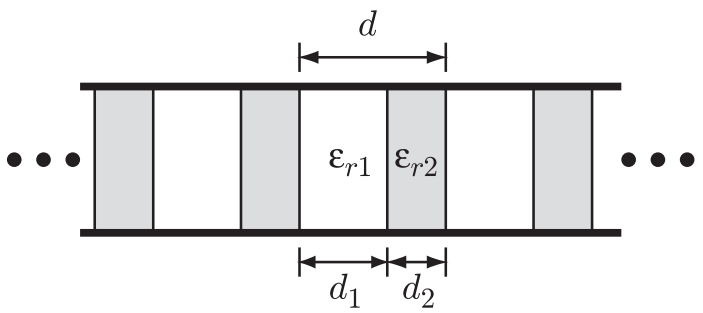

Fig. A. 1 1-D infinite periodic structure in H-plane waveguide component. 


$$
\begin{aligned}
\frac{A_{11}+A_{22}}{2}= & \frac{1+p}{2} \cos \left(\beta_{1} d_{1}+\beta_{2} d_{2}\right) \\
& +\frac{1-p}{2} \cos \left(\beta_{1} d_{1}-\beta_{2} d_{2}\right)
\end{aligned}
$$

where

$$
p=\frac{1}{2}\left(\frac{\beta_{2}}{\beta_{1}}+\frac{\beta_{1}}{\beta_{2}}\right)
$$

Here $\beta_{i}$ and $d_{i}(i=1,2)$ are the propagation constant and length of a waveguide section with relative permittivity $\varepsilon_{r i}$. Assuming that $\beta_{1}^{(c)} d_{1}+\beta_{2}^{(c)} d_{2}=\pi$ is approximately satisfied at the central frequency $f^{(c)}$ of the stopband, we can find the attenuation constant $\alpha$ as a function of frequency against a periodic length $d\left(d=d_{1}+d_{2}\right)$ from (A-1).

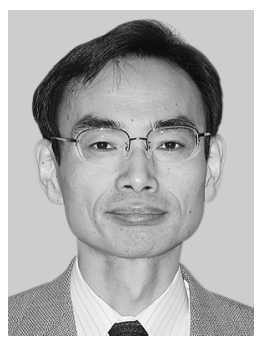

Koichi Hirayama received the B.S., M.S., and Ph.D. degrees in electronic engineering from Hokkaido University, Sapporo, Japan, in 1984, 1986, and 1989, respectively. In 1989, he joined the Department of Electronic Engineering, Kushiro National College of Technology, Kushiro, Japan. In 1992, he became an Associate Professor of Electronic Engineering at Kitami Institute of Technology, Kitami, Japan, and in 2004 he became a Professor. He has been interested in the analysis and optimal design of electromagnetic and optical waveguides. Dr. Hirayama is a senior member of IEEE and a member of the Japan Society of Applied Physics.

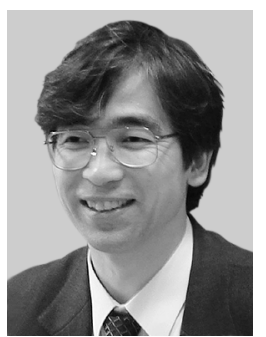

Yasuhide Tsuji received the B.S., M.S., and $\mathrm{Ph} . \mathrm{D}$. degrees in electronic engineering from Hokkaido University, Sapporo, Japan, in 1991 1993, and 1996, respectively. In 1996, he joined the Department of Applied Electronic Engineering, Hokkaido Institute of Technology, Sapporo, Japan. From 1997 to 2004, he was an Associate Professor of Electronics and Information Engineering at Hokkaido University. Since 2004, he has been an Associate Professor of Electronic Engineering at Kitami Institute of Technology, Kitami, Japan. He has been interested in wave electronics. Dr. Tsuji is a member of the Japan Society of Applied Physics and IEEE. In 1997 and 1999, he was awarded the Excellent Paper Award from IEICE.

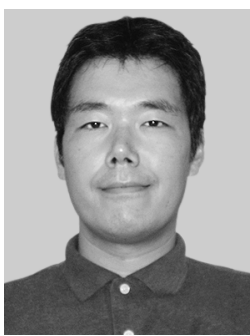

Shintaro Yamasaki earned his Bachelor of Engineering Degree in Precision Engineering from Kyoto University in 1997 and his Master Degree in Precision Engineering from Kyoto University in 1999. He also received his Doctor of Philosophy Degree in Aerospace Engineering from Kyoto University in 2009. He is now an associate professor in the College of Systems Engineering and Science at Shibaura Institute of Technology. His research interests include structural optimization of mechanical structures,

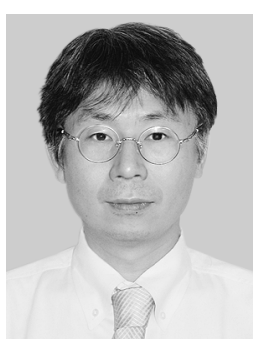

Shinji Nishiwaki earned his Bachelor of Engineering Degree in Precision Engineering from Kyoto University in 1986 and his Master Degree in Precision Engineering from Kyoto University in 1988. He also received his Doctor of Philosophy Degree in Mechanical Engineering from the University of Michigan in 1998. He is now a professor in the Graduate School of Engineering at Kyoto University. His research interests include structural optimization of mechanical structures, sensors, and actuators, and 